\title{
CAUCHY-GREEN TYPE FORMULAE IN CLIFFORD ANALYSIS
}

\author{
JOHN RYAN
}

\begin{abstract}
A Cauchy integral formula is constructed for solutions to the polynomial Dirac equation $\left(D^{k}+\sum_{m=0}^{k-1} b_{m} D^{m}\right) f=0$, where each $b_{m}$ is a complex number, $D$ is the Dirac operator in $R^{n}$, and $f$ is defined on a domain in $R^{n}$ and takes values in a complex Clifford algebra. Some basic properties for the solutions to this equation, arising from the integral formula, are described, including an approximation theorem. We also introduce a Bergman kernel for square integrable solutions to $(D+\lambda) f=0$ over bounded domains with piecewise $C^{1}$, or Lipschitz, boundary.
\end{abstract}

\section{INTRODUCTION}

It is well understood $[1,3,4]$ that solutions to the Dirac equation, $D f=0$, in $R^{n}$ can be described by a Cauchy integral formula. Here, $D$ stands for the homogeneous Dirac operator $\sum_{j=1}^{n} e_{j} \partial / \partial x_{j}$, and the $e_{j}$ 's are the generators of a real Clifford algebra $A_{n}$. As $D^{2}=-\Delta$, the negative Laplacian over $R^{n}$, it may easily be shown (see [8]) that Green's formula for harmonic functions can be modified via Clifford algebras to more closely resemble a Cauchy integral formula.

In [12], $X$. Zhenyuan shows that solutions to the inhomogeneous Dirac equation $(D+\lambda) f=0$, with $\lambda \in \mathbf{C}$, also possess a Cauchy integral formula, with a Cauchy kernel $E_{-\lambda}(x-y)$. A similar kernel for this equation is also produced for the case $n=3$ by Gürlebeck and Sprössig [4, Chapter 4] and is used to describe boundary value problems for Helmholtz' equation $\Delta h=\lambda^{2} h$. Using the kernel $E_{\lambda}$, produced in [12], these results automatically generalize to the cases $n>3$. Clifford algebras have also been used by Mitrea [6] to study boundary value problems and associated $H^{p}$ spaces for the Helmholtz equation over nonsmooth domains.

In [11], Sommen and $\mathrm{X}$. Zhenyuan construct a Cauchy kernel for $D-\lambda$ as a power series $\sum_{m=1}^{\infty} a_{m} G_{m}(x)$, where $a_{m} \in R$, and $G_{m}(x)$ is a fundamental solution to the operator $D^{m}$. In this paper, we show that this method of construction generalizes to allow us to obtain Cauchy kernels and CauchyGreen type integral formulae for solutions to each polynomial equation $\left(D^{k}+\sum_{m=0}^{k-1} b_{m} D^{m}\right) h=0$, with $b_{m} \in \mathbf{C}$. It automatically follows that many existing results in Clifford analysis extend to the context described here; for instance, the approximation theorems described in [1, Chapter 3] automatically

Received by the editors October 14, 1993 and, in revised form, December 13, 1993; originally communicated to the Proceedings of the AMS by Palle E. T. Jorgensen.

1991 Mathematics Subject Classification. Primary 30G35. 
generalize. We briefly illustrate some of these points. We also show that the results given here give improved information over that given in [9] on the holomorphic continuation of solutions to $\left(D^{k}+\sum_{m=0}^{k-1} b_{m} D^{m}\right) f=0$, and building upon results presented in [10], we produce a Bergman kernel for square integrable solutions to $(D-\lambda) f=0$ over arbitrary bounded domains with piecewise $C^{1}$, or Lipschitz, boundary.

\section{Preliminaries}

We shall consider the real, $2^{n}$-dimensional Clifford algebra, $A_{n}$, generated from $R^{n}$ equipped with the negative definite inner product. Consequently, if $\left\{e_{j}\right\}_{j=1}^{n}$ is an orthonormal basis for $R^{n}$, then $A_{n}$ has basis elements

$$
1, e_{1}, \ldots, e_{n}, \ldots, e_{j_{1}} \cdots e_{j_{r}}, \ldots, e_{1} \cdots e_{n},
$$

where 1 is the unit of the algebra, $j_{1}<\cdots<j_{r}, 1 \leq r \leq n$, and the elements $e_{1}, \ldots, e_{n}$ satisfy the anticommutation relationship

$$
e_{j} e_{k}+e_{k} e_{j}=-2 \delta_{j k}, \quad 1 \leq j, k \leq n,
$$

with $\delta_{j, k}$ the Kronecker delta.

The Euclidean Dirac operator $D$ is the differential operator

$$
\sum_{j=1}^{n} e_{j} \frac{\partial}{\partial x_{j}} \text {. }
$$

It may easily be observed that $D^{2}=-\Delta_{n}$, where $\Delta_{n}$ is the Laplacian in $R^{n}$.

We shall use the complexification $A_{n}(\mathbf{C})$, of $A_{n}$.

Definition. Suppose $U$ is a domain in $R^{n}$, and $f: U \rightarrow A_{n}(\mathbf{C})$ is a $C^{1}$ function satisfying $D f=0$. Then $f$ is called a left-monogenic function. If the $C^{1}$-function $h: U \rightarrow A_{n}(\mathbf{C})$ satisfies

$$
h D=\sum_{j=1}^{n} \frac{\partial h}{\partial x_{j}} e_{j}=0,
$$

then $h$ is called a right-monogenic function.

An example of a function which is both left- and right-monogenic is the function $G_{1}(x)=\left(1 / w_{n}\right) x /\|x\|^{n}$ defined on $R^{n} \backslash\{0\}$. Here, $w_{n}$ denotes the surface area of the unit sphere in $R^{n}$. It should also be noted that we are assuming that $n>1$.

The function theory associated to monogenic functions and the applications of this function theory have been pursued by many authors (see, for instance, [1-6, 8-12]). For $n>2$, the function $G_{1}(x)$ is a generalization of the Cauchy kernel from one-variable complex analysis. In particular, from Stokes' theorem, the homogeneity of $G_{1}(x)$, and the fact that $x^{2}=-\|x\|^{2}$ for each $x \in R^{n}$, we have

Theorem 1 (Cauchy's integral formula). Suppose that $f: U \rightarrow A_{n}(\mathbf{C})$ is leftmonogenic, and $M$ is a closed, bounded region in $U$ with piecewise $C^{1}$ (or Lipschitz continuous) boundary. Suppose also that $x_{0} \in M^{\circ}$, where $M^{\circ}$ denotes the interior of $M$. Then

$$
f\left(x_{0}\right)=\int_{\partial M} G_{1}\left(x-x_{0}\right) n(x) f(x) d \partial M,
$$


where $n(x)$ is the unit vector outward normal to $M$ at $x$, and dəM denotes the usual Lebesgue measure on $\partial M$.

This classical result was established for the case $n=3$ by Dixon [3] in 1904 and has been reintroduced and proved many times since.

Besides the Cauchy kernel $G_{1}(x)$, we also have the kernels $G_{k}(x)$, where

$$
\begin{gathered}
G_{2 l+1}(x)=A_{2 l+1} \frac{x}{\|x\|^{n-2 l}}, \quad l=0,1, \ldots, \\
G_{2 l}(x)=A_{2 l} \frac{1}{\|x\|^{n-2 l}}, \quad l=1, \ldots,
\end{gathered}
$$

when $n$ is odd, and

$$
\begin{gathered}
G_{2 l+1}(x)=A_{2 l+1}^{\prime} \frac{x}{\|x\|^{n-2 l}}, \quad l=0,1, \ldots, \frac{n}{2}-1, \\
G_{2 l}(x)=A_{2 l}^{\prime} \frac{1}{\|x\|^{n-2 l}}, \quad l=1, \ldots, \frac{n-2}{2}, \\
G_{k}(x)=B_{k} x^{k-n}+C_{k} x^{k-n} \ln \|x\|, \quad \text { for } k \geq n,
\end{gathered}
$$

when $n$ is even. Moreover, the real coefficients $A_{k}, A_{k}^{\prime}, B_{k}$, and $C_{k}$ are chosen so that $D G_{k}=G_{k-1}$. It follows that $G_{k} D=G_{k-1}$, and $D^{k} G_{k}=0$. Using the functions $G_{k}$, we have from Stokes' theorem, Theorem 1 and simple homogeneity arguments.

Theorem 2 [9]. Suppose that $g: U \rightarrow A_{n}(\mathbf{C})$ satisfies the equation $D^{k} g=0$. Suppose also that $M \subseteq U$ is as in Theorem 1 , and $x_{0} \in M^{\circ}$. Then

$$
g\left(x_{0}\right)=\int_{\partial M} \sum_{j=1}^{k}(-1)^{j-1} G_{j}\left(x-x_{0}\right) n(x) D^{j-1} g(x) d \partial M .
$$

In [11] it is shown that for each $\lambda \in \mathbf{C}$, the series $\sum_{l=1}^{\infty} \lambda^{l-1} G_{l}\left(x-x_{0}\right)$ is well defined on $R^{n} \backslash\left\{x_{0}\right\}$, and $(D-\lambda) E_{-\lambda}\left(x-x_{0}\right)=E_{-\lambda}\left(x-x_{0}\right)(D-\lambda)=0$, where

$$
E_{-\lambda}\left(x-x_{0}\right)=\sum_{l=1}^{\infty} \lambda^{l-1} G_{l}\left(x-x_{0}\right) .
$$

The convergence of the series $\sum_{l=1}^{\infty} \lambda^{l-1} G_{l}\left(x-x_{0}\right)$ follows easily in the case $n$ odd by simply checking convergence for the series $\sum_{l=n+1}^{\infty} \lambda^{l-1} G_{l}\left(x-x_{0}\right)$. It is then a reasonably straightforward exercise to check for convergence for the cases $n$ even.

Proposition 1. Suppose that $A(x)$ is a $C^{1}$-function defined on $U$, and $f, g$ : $U \rightarrow A_{n}(\mathbf{C})$. Furthermore, $D f(x)+A(x) f(x)=0$, while $g(x) D-g(x) A(x)=$ 0 . Then we have

$$
\int_{\partial M} g(x) n(x) f(x) d \partial M=0,
$$

for each closed, bounded region $M \subseteq U$, with piecewise $C^{1}$, or Lipschitz, boundary.

As $E_{-\lambda}\left(x-x_{0}\right)=G_{1}\left(x-x_{0}\right) Q_{\lambda}\left(x-x_{0}\right)$, where $Q_{\lambda}(x)$ is a bounded function, and $Q_{\lambda}(0)=1$, it follows from Proposition 1 that one may deduce 
Theorem 3 [12]. Suppose that $g: U \rightarrow A_{n}(\mathbf{C})$ satisfies $(D+\lambda) g=0$, and $M \subseteq U$ is as in Theorem 1. Then we have

$$
g\left(x_{0}\right)=\int_{\partial M} E_{-\lambda}\left(x-x_{0}\right) n(x) g(x) d \partial M,
$$

for each $x_{0} \in M^{\circ}$.

A similar result holds for solutions to the equation

$$
h(x) D+\lambda h(x)=0 .
$$

From Theorem 3 it follows that many existing results which hold for solutions to $D f=0$ carry over to solutions to $D g+\lambda g=0$. For instance, via Stokes' theorem we have the following Pompeiu representation for $C^{1}$-functions.

Theorem 4. Suppose that $h: U \rightarrow A_{n}(\mathbf{C})$ is a $C^{1}$-function, and $M$ is a bounded, closed region in $U$, with piecewise $C^{1}$, or Lipschitz, boundary. Then we have that

$$
h\left(x_{0}\right)=\int_{\partial M} E_{-\lambda}\left(x-x_{0}\right) n(x) h(x) d \partial M-\int_{M} E_{-\lambda}\left(x-x_{0}\right)(D+\lambda) h(x) d x^{n},
$$

for each $x_{0} \in M^{\circ}$.

We also have

Proposition 2. Suppose that $U$ is a bounded domain, and $h: U \rightarrow A_{n}$ is a bounded, $C^{1}$-function. Then the integral

$$
\int_{U} E_{-\lambda}\left(x-x_{0}\right) h(x) d x^{n}
$$

is well defined on $U$ and gives a $C^{1}$-function.

Outline Proof. As $E_{-\lambda}\left(x-x_{0}\right)=G_{1}\left(x-x_{0}\right) Q_{\lambda}\left(x-x_{0}\right)$, where $Q_{\lambda}(x)$ is a locally bounded, real analytic function on $R^{n}$, it follows that the integral (1) is well defined.

Suppose now that $u \in S^{n-1}$, the unit sphere in $R^{n}$, and $\frac{\partial}{\partial u}$ denotes the partial differentiation in the direction of $u$. Suppose also that $B_{u}\left(x_{0}\right)$ is a box lying in $U$ with its center at $x_{0}$ and with a face normal to $u$. Then

$$
\begin{aligned}
\lim _{\delta \rightarrow 0} \frac{1}{\delta} & \left(\int_{U}\left(E_{-\lambda}\left(x-x_{0}\right)-E_{-\lambda}\left(x-x_{0}-\delta u\right)\right) h(x) d x^{n}\right) \\
= & \int_{U \backslash B_{u}\left(x_{0}\right)} \frac{\partial E_{-\lambda}}{\partial u}\left(x-x_{0}\right) h(x) d x^{n}+\int_{B_{u}\left(x_{0}\right)} E_{-\lambda}\left(x-x_{0}\right) \frac{\partial h}{\partial u}(x) d x^{n} \\
& +\int_{B_{1}\left(x_{0}\right)} E_{-\lambda}\left(x-x_{0}\right) h(x) d B_{1}\left(x_{0}\right)-\int_{B_{2}\left(x_{0}\right)} E_{-\lambda}\left(x-x_{0}\right) h(n) d B_{2}\left(x_{0}\right),
\end{aligned}
$$

where $B_{1}\left(x_{0}\right)$ is the face of $B_{u}\left(x_{0}\right)$ with $u$ as an outward-pointing normal vector, while $B_{2}\left(x_{0}\right)$ is the face of $B_{u}\left(x_{0}\right)$ with $-u$ as an outward-pointing normal vector.

As a consequence of Proposition 2, we have 
Theorem 5. Suppose that $U$ is a bounded domain and $h: U \rightarrow A_{n}(\mathbf{C})$ is a bounded $C^{1}$-function. Then

$$
(D-\lambda) \int_{U} E_{-\lambda}\left(x-x_{0}\right) h(x) d x^{n}=-h\left(x_{0}\right) .
$$

Using the fact that $E_{-\lambda}\left(x-x_{0}\right)=Q_{\lambda}\left(x-x_{0}\right) G_{1}\left(x-x_{0}\right)$, with $Q_{\lambda}(x)$ a locally bounded function, and $Q_{\lambda}(0)=1$, it is straightforward to show that

$$
\left\|f\left(x_{0}\right)\right\| \leq \frac{C\left(A\left(X_{0}, r_{1}, r_{2}\right)\right)}{r_{R}^{n-1}\left(r_{1}-r_{2}\right)} \int_{A\left(x_{0}, r_{1}, r_{2}\right)}\|f(x)\| d x^{n},
$$

where $\|J\|=\left(\left|j_{0}\right|^{2}+\cdots+\left|j_{1 \cdots n}\right|^{2}\right)^{1 / 2}$ for each $J=J_{0}+\cdots+j_{1 \cdots n} e_{1} \cdots e_{n} \in A_{n}(\mathbf{C})$, where $j_{0}, j_{1 \cdots n} \in \mathbf{C}$ while $r_{1}, r_{2} \in R^{+}$with $r_{2}<r_{1}$, and

$$
A\left(x_{0}, r_{1}, r_{2}\right)=\left\{x \in R^{n}: r_{2}<\left\|x-x_{0}\right\|<r_{1}\right\} .
$$

Moreover, the ball $B\left(x_{0}, r_{1}\right)$ lies in $U$, and $C\left(A\left(x_{0}, r_{1}, r_{2}\right)\right) \in R^{+}$. From Hölder's inequality, it now follows that the right $A_{n}(\mathbf{C})$-module of $L^{p}$-integrable functions, $1 \leq p \leq \infty$, on $U$, and satisfying $D f+\lambda f=0$, is complete. This is a complete analogy to the case $\lambda=0$ described in [4]. This result is described for the case $n=3$ in [4, Chapter 4]. The special case $p=2$ is also described in [2].

\section{Polynomial Dirac eQuations}

In this section, we study some properties of solutions to the polynomial Dirac equation $\left(D^{k}+\sum_{m=0}^{k-1} b_{m} D^{m}\right) g=0$, where each $b_{m} \in \mathbf{C}$. In the case where $k=2$ and $b_{1}=0$, this equation becomes Helmholtz' equation. We shall begin by constructing a solution to the general equation $\left(D^{k}+\sum_{m=0}^{k-1} b_{m} D^{m}\right) g=0$ from the function $G_{k}(x)$. If at least one of the coefficients $b_{m}$ is nonzero, then the function $G_{k}(x)$ is not annihilated by the polynomial Dirac operator $D^{k}+\sum_{m=0}^{k-1} b_{m} D^{m}$. In this case, we may apply the operator $\sum_{m=0}^{k-1} b_{m} D^{m}$ to $G_{k}(x)$ to obtain $\sum_{m=0}^{k-1} b_{m} G_{k-m}(x)=-D^{k} \sum_{m=0}^{k-1} b_{m} G_{2 k-m}(x)$. Upon applying the operator $\sum_{m=0}^{k-1} b_{m} D^{m}$ to the function $\sum_{m=0}^{k-1} b_{m} G_{2 k-m}(x)$, we would obtain the first few terms in the formal series

$$
\sum_{p=0}^{\infty}(-1)^{p}\left(\sum_{m=0}^{k-1} b_{m} D^{m}\right)^{p} G_{k(1+p)}(x) .
$$

For any domain on which this series converges, we have a solution to the equation $\left(D^{k}+\sum_{m=0}^{k-1} b_{m} D^{m}\right) g=0$.

The method of constructing the formal series (2) is a direct analogue of a standard recursive method for constructing solutions to ordinary differential equations. We first establish convergence of the formal series (2) for the cases $n$ odd. The case of $n$ even is complicated slightly by the presence of the log function in $G_{q}(x)$ for $q \geq n$. A simple inspection of the series

$$
\sum_{p=0}^{\infty}(-1)^{p} \sum_{m_{p}=0}^{k-1} \cdots \sum_{m_{1}=0}^{k-1} b_{m_{p}} \cdots b_{m_{1}} G_{k(p+1)-m_{p} \cdots m_{1}}(x)
$$

reveals that for some integer $m(k)$ the functions $G_{k(p+1)-m_{p}-\cdots-m_{1}}(x)$ each have a positive degree of homogeneity for $p+1 \geq m(k)$. It follows that in 
order to test the convergence of (3), it is sufficient to test convergence of the series

$$
\sum_{p=0}^{\infty}(-1)^{p} \sum_{m_{p}=0}^{k-1} \cdots \sum_{m_{1}=0}^{k-1} b_{m_{p}} \cdots b_{m_{1}} G_{k(p+m(k))-m_{p}-\cdots-m_{1}}(x) .
$$

As $D\|x\|^{2 q-1}=(2 q-1) x\|x\|^{2 q-3}, D x\|x\|^{2 q-1}=(-2 q-n+1)\|x\|^{2 z-1}$, and $2 q+n-1>2 q$, we have that $\left\|G_{L+n}(x)\right\| \leq c(n) \frac{1}{L !}\|x\|^{L}$, for some constant $c(n) \in R^{+}$. Convergence for series (4) on $R^{n}$ for the special case $b_{k-1}=\cdots=$ $b_{1}=0$ and $b_{0}=\lambda \in \mathbf{C}$ now follows quite easily upon comparing (4) with the exponential series $\sum_{p=0}^{\infty} \frac{1}{p !}|\lambda|^{p}\|x\|^{p}$. Also, the convergence of (4) on $R^{n}$ for the special case $b_{0}=0, b_{1}=1, b_{j}=0, j=2, \ldots, k-1$, follows by comparison with the exponential series. Convergence on $R^{n}$ for other special cases of the series (4) follows similarly. To establish convergence, on $R^{n}$, of the series (4) in the general case, we may first place

$$
b=\sup \left\{1,\left|b_{0}\right|, \ldots,\left\{b_{k-1}\right\}\right\} \text {. }
$$

It may now be observed that $\left|b_{m_{1}} \cdots b_{m_{p}}\right| \leq b^{L}$, where $L=k(p+m(k))-m_{1}-$ $\cdots-m_{p}$. It follows that

$$
\begin{aligned}
& \left\|\sum_{p=0}^{\infty}(-1)^{p} \sum_{m_{p}=0}^{k-1} \cdots \sum_{m_{1}=0}^{k-1} b_{m_{p}} \cdots b_{m_{1}} G_{k(p+m(k))-m_{p}-\cdots-m_{1}}(x)\right\| \\
& \quad \leq c(n) \sum_{L=0}^{\infty} \frac{A(L)}{L !} b^{L}\|x\|^{L}
\end{aligned}
$$

where $A(L)$ denotes the number of occurrences of the term $G_{L}(x)$ in (4).

We now need to obtain appropriate estimates for the coefficient $A(L)$. It may be observed that $A(L) \leq B(L)$, where $B(L)$ is the order of the set

$$
\begin{aligned}
R(L)=\left\{\left(a_{1}, \ldots, a_{p}\right): p \in \mathbf{N}, a_{j}=1, \ldots, k\right. \\
\text { with } \left.1 \leq j \leq p, \text { and } a_{1}+\cdots+a_{p}=L\right\} .
\end{aligned}
$$

To estimate $B(L)$, we first obtain an estimate for the order of the set

$$
\begin{aligned}
Q(L)=\left\{\left(a_{1}, \ldots, a_{p}\right): p\right. & \in \mathbf{N}, a_{j} \in\{1, \ldots, k\} \\
& \text { with } \left.1 \leq j \leq n, a_{1}+\cdots+a_{p}=L, \text { and } a_{1} \leq a_{2} \leq \cdots \leq a_{p}\right\} .
\end{aligned}
$$

There is precisely one element in $Q(L)$ whose entries are all 1 . There are at most $\left[\frac{L}{2}\right]$ elements in $Q(L)$ whose entries are either 1 or 2 . Moreover, there are at most $\left[\frac{L}{k}\right]$ elements whose entries are either 1 or $k$.

Consequently, there are most $1+\left[\frac{L}{2}\right]+\cdots+\left[\frac{L}{k}\right]$ elements in $Q(L)$ whose entries are either 1 or $j$, with $j=2, \ldots, k$. Now, $1+\left[\frac{L}{2}\right]+\cdots+\left[\frac{L}{k}\right] \leq$ $1+L\left(1+\frac{1}{2}+\cdots+\frac{1}{k}\right)$. Similarly, one can show that the number of elements in $Q(L)$, whose entries contain only $1, j$, and $k$, with $j \neq k$, and with at least one $j$ and at least one $k$, is bounded by $L^{2}\left(1+\frac{1}{2}+\cdots+\frac{1}{k}\right)^{2}$. Continuing in 
this way, we see that the order of the set $Q(L)$ is bounded above by

$$
\begin{aligned}
1+ & L\left(1+\cdots+\frac{1}{k}\right)+\cdots+L^{k}\left(1+\cdots+\frac{1}{k}\right)^{k} \\
& \leq\left(1+\frac{1}{2}+\cdots+\frac{1}{k}\right)^{k}\left(1+L+\cdots+L^{k}\right) \\
& =\left(1+\frac{1}{2}+\cdots+\frac{1}{k}\right)^{k} \frac{\left(L^{k+1}-1\right)}{(L-1)} \leq 2\left(1+\cdots+\frac{1}{k}\right)^{k} L^{k} .
\end{aligned}
$$

In order to obtain an upper bound for the order of the set $R(L)$ we can multiply the estimate $2\left(1+\cdots+\frac{1}{k}\right)^{k} L^{k}$ by the maximum number, $D(L)$, of permutations possible for any entry $a_{1}, \ldots, a_{p}$, where $\left(a_{1}, \ldots, a_{p}\right) \in Q(L)$.

To obtain an estimate for $D(L)$, we first take an element

$$
(1, \ldots, 1,2, \ldots, 2,3, \ldots, k, \ldots, k) \in Q(L),
$$

where 1 appears $q_{1}$ times, 2 appears $q_{2}$ times, ..., and $k$ appears $q_{k}$ times. So, $q_{1}+2 q_{2}+\cdots+k q_{k}=L$. The number of possible permutations of these entries without repetition is

$$
\frac{\left(L-q_{2}-2 q_{3}-\cdots-(k-1) q_{k}\right) !}{q_{1} ! \cdots q_{k} !}=\frac{\left(q_{1}+q_{2}+\cdots+q_{k}\right) !}{q_{1} ! \cdots q_{k} !} .
$$

Also, $q_{1}+\cdots+q_{k} \leq L$. As $q_{1}+\cdots+q_{k} \leq L$, we have that $D(L) \leq \frac{L !}{q_{1} ! \cdots q_{k} !} \leq \frac{L !}{q_{j} !}$ for $j=1, \ldots, k$. As $q_{1}+2 q_{2}+\cdots+k q_{k}=L$, we have that at least one $q_{j} \geq\left[\frac{L}{k^{2}}\right]$ for $L \geq k^{2}$. Consequently, $D(L) \leq \frac{L !}{\left[L / k^{2}\right] !}$. It follows that $A(L) \leq$ $2\left(1+\cdots+\frac{1}{k}\right)^{k} L^{k} \frac{L !}{\left[L / k^{2}\right] !}$ for $L$ large enough. So, the series on the right-hand side of (5) is dominated by the first $k^{2}-1$ terms and the series

$$
c(n) \sum_{L=k^{2}}^{\infty} 2\left(1+\cdots+\frac{1}{k}\right)^{k} \frac{L^{k}}{\left[\frac{L}{k^{2}}\right] !} b^{L}\|x\|^{L} .
$$

By placing $L=m k^{2}+L \bmod k^{2}$ in the previous series and applying the ratio test to the resulting $k^{2}-1$ series in $m$, it follows that each series converges on $R^{n}$. Consequently, the series (2) gives a solution to the equation $\left(D^{k}+\sum_{m=0}^{k-1} b_{m} D^{m}\right) h=0$ on $R^{n} \backslash\{0\}$ whenever $n$ is odd.

We now turn to the case $n$ even. This case follows similar lines to the odd-dimensional case. Again, we have that after finitely many terms, series (2) contains only $G_{L}(x)$ 's, which do not have negative degrees of homogeneity. For such a $G_{L}(x)$, we have that

$$
\left\|G_{L}(x)\right\| \leq c^{\prime}(n) \frac{2}{L !}\left(\|x\|^{L}+\|x\|^{L} \ln \|x\|\right),
$$

for some $c^{\prime}(n) \in R^{+}$. Consequently, convergence of series (2) on $R^{n} \backslash\{0\}$ when $n$ is even follows by similar arguments to those given for the case $n$ odd.

It follows that we have deduced

Theorem 6. Series (2) converges on $R^{n} \backslash\{0\}$, for each $n$, and gives a solution to the equation $\left(D^{k}+\sum_{m=0}^{k-1} b_{m} D^{m}\right) h=0$ on this domain.

We shall now denote the function given in expression (2) by $E_{b_{0}, \ldots, b_{k-1}}(x)$. From this function we can construct other solutions to the equation $\left(D^{k}+\sum_{m=0}^{k-1} b_{m} D^{m}\right) h=0$ as follows: 
Proposition 3. Suppose that $\mu$ is an $A_{n}(\mathbf{C})$-valued measure on $R^{n}$, with compact support $[\mu]$. Then $\int_{[\mu]} E_{b_{0}, \ldots, b_{k-1}}(x-y) d \mu(y)$ gives a solution to $\left(D^{k}+\sum_{m=0}^{k-1} b_{m} D^{m}\right) h=0$ on $R^{n} \backslash[\mu]$.

It may also be observed from the construction of $E_{b_{0}, \ldots, b_{k-1}}(x)$ that this function also satisfies the equation $h D^{k}+\sum_{m=0}^{k-1} b_{m} h D^{m}=0$. Consequently, for each $A_{n}(\mathbf{C})$-valued measure $\mu$ with compact support [ $\mu$ ] on $R^{n}$, we have that $\int_{[\mu]} d \mu(y) E_{b_{0}, \ldots, b_{k-1}}(x-y)$ gives a solution to $h D^{k}+\sum_{m=0}^{k-1} b_{m} h D^{m}=0$ on $R^{n} \backslash[\mu]$. We now show that the kernel $E_{b_{0}, \ldots, b_{k-1}}(x-y)$ acts as a Cauchy-Green kernel for solutions to the equation $\left(D^{k}+\sum_{m=0}^{k-1}(-1)^{k-m} b_{m} D^{m}\right) h=0$.

Theorem 7. Suppose that $f: U \rightarrow A_{n}(\mathbf{C})$ satisfies the equation

$$
\left(D^{k}+\sum_{m=0}^{k-1}(-1)^{k-m} b_{m} D^{m}\right) f=0,
$$

and $M$ is a closed bounded region in $U$, with piecewise $C^{1}$ (or Lipschitz) boundary. Then for each $x_{0} \in M^{\circ}$, we have

(6)

$$
\begin{aligned}
f\left(x_{0}\right)= & \int_{\partial M}\left(\sum_{j=0}^{k-1}(-1)^{j}\left(E_{b_{0}, \ldots, b_{k-1}}\left(x-x_{0}\right) D^{k-1-j}\right) n(x) D^{j} f(x)\right) \\
& +\sum_{m=1}^{k-1} b_{m} \sum_{j=0}^{m-1}(-1)^{j}\left(E_{b_{0}, \ldots, b_{k-1}}\left(x-x_{0}\right) D^{m-1-j}\right) n(x) D^{j} f(x) d \partial M .
\end{aligned}
$$

Outline Proof. The proof follows from Stokes' theorem, and upon noting that $E_{b_{0}, \ldots, b_{k-1}}\left(x-x_{0}\right) D^{k-1}=G\left(x-x_{0}\right) K_{b_{1} \cdots b_{k-1}}\left(x-x_{0}\right)$, where $K_{b_{0}, \ldots, b_{k-1}}(0)=1$, and $K_{b_{0} \cdots b_{k-1}}$ is continuous on $R^{n}$.

Having obtained the Cauchy-Green integral formula (6), it follows that many existing results obtained for solutions to the Dirac equation, or Laplace equation, carry through to the context described here. For instance, by similar arguments to those given in [7, Chapter 13], or [1, Chapter 3], we have the following approximation theorem:

Theorem 8. Suppose that $J$ is a compact set in $R^{n}$, and $R^{n} \backslash J$ is connected. Suppose also that $\Omega$ is a domain in $R^{n}$ containing $J$. Then, for each function $h: \Omega \rightarrow A_{n}(\mathbf{C})$ satisfying

$$
\left(D^{k}+\sum_{m=0}^{k-1}(-1)^{k-m} b_{m} D^{m}\right) h=0,
$$

and for each ball $B(0, R)$ with $\Omega \subseteq B(0, R)$, there is for each $\varepsilon>0$ a function $f: B(0, R) \rightarrow A_{n}(\mathbf{C})$ satisfying

$$
\left(D^{k}+\sum_{m=0}^{k-1}(-1)^{k-m} b_{m} D^{m}\right) f=0
$$

and

$$
\sup _{x \in J}\|f(x)-h(x)\|<\varepsilon
$$


Following the sequential argument given in [1, Theorem 18.4], we may, in the previous theorem, replace $B(0, R)$ by $R^{n}$, and assume that $f$ is defined on all $R^{n}$. Following [7, Chapter 13], and [1, Chapter 3], we may also drop the assumption, in Theorem 8 , that the set $R^{n} \backslash J$ is connected. We also have the following Pompeiu representation formula:

Theorem 9. Suppose that $g: U \rightarrow A_{n}(\mathbf{C})$ is a $C^{k}$-function, and $M$ is a closed bounded region in $U$ with Lipschitz boundary. Then for each $x_{0} \in M^{\circ}$, we have

$$
\begin{aligned}
g\left(x_{0}\right)= & \int_{\partial M}\left(\sum_{j=0}^{k-1}(-1)^{j}\left(E_{b_{0}, \ldots, b_{k-1}}\left(x-x_{0}\right) D^{k-1-j}\right) n(x) D^{j} f(x)\right) \\
& +\sum_{m=1}^{k-1} b_{m} \sum_{j=0}^{m-1}(-1)^{j}\left(E_{b_{0}, \ldots, b_{k-1}}\left(x-x_{0}\right) D^{m-1-j}\right) n(x) D^{j} j(x) d \partial M \\
& +\int_{M} E_{b_{0}, \ldots, b_{k-1}}\left(x-x_{0}\right)\left(D^{k}+\sum_{m=1}^{k-1}(-1)^{k-m} b_{m} D^{m}\right) g(x) d x^{n} .
\end{aligned}
$$

If we assume that the function $g(x)$ appearing in Theorem 9 has compact support, then expression (7) becomes

$$
g\left(x_{0}\right)=\int_{R^{n}} E_{b_{0}, \ldots, b_{n-1}}\left(x-x_{0}\right)\left(D^{k}+\sum_{m=0}^{k-1}(-1)^{k-m} b_{m} D^{m}\right) g(x) d x^{n} .
$$

We also have that whenever $U$ is a bounded domain and $g: U \rightarrow A_{n}(\mathbf{C})$ is a $C^{k}$-function, then

$$
\left(D^{k}+\sum_{m=0}^{k-1} b_{m} D^{m}\right) \int_{U} E_{b_{0}, \ldots, b_{k-1}}\left(x-x_{0}\right) g(x) d x^{n}=g\left(x_{0}\right)
$$

for each $x_{0} \in U$. When $g$ has compact support, this identity can be replaced by

$$
\left(D^{k}+\sum_{m=0}^{k-1} b_{m} D^{m}\right) \int_{R^{n}} E_{b_{0}, \ldots, b_{k-1}}\left(x-x_{0}\right) g(x) d x^{n}=g\left(x_{0}\right) .
$$

Expressions (8) and (9) show us that the convolution operator $E_{b_{0}, \ldots, b_{k-1}} \star_{R^{n}}$ is a left inverse for the differential operator $D^{k}+\sum_{m=0}^{k-1}(-1)^{k-m} b_{m} D^{m}$, while the differential operator $D^{k}+\sum_{m=0}^{k-1} b_{m} D^{m}$ is a left inverse for the convolution

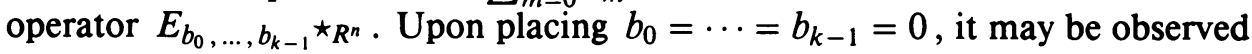
that the convolution operator $G_{k} \star_{R^{n}}$, when acting upon $A_{n}(\mathbf{C})$-valued $C^{\infty}$. functions with compact support, is a left inverse for the operator $D^{k}$. Suppose now that $\psi: R^{n} \rightarrow A_{n}(\mathbf{C})$ is a $C^{\infty}$-function with compact support. Upon taking the Fourier transform of

$$
\left(D^{k}+\sum_{m=0}^{k-1}(-1)^{k-m} b_{m} D^{m}\right) \psi
$$


we obtain

$$
\begin{aligned}
& \lambda(n) \int_{R^{n}} e^{i\langle x, \xi\rangle}\left(D^{k}+\sum_{m=0}^{k-1}(-1)^{k-m} b_{m} D^{m}\right) \psi(x) d x^{n} \\
& \quad=\lambda(n)(-1)^{k}\left((i \xi)^{k}+\sum_{m=0}^{k-1} b_{m}(i \xi)^{m}\right) \int_{R^{n}} e^{i\langle x, \xi\rangle} \psi(x) d x^{n},
\end{aligned}
$$

where $\lambda(n)$ is a normalizing constant.

It may be observed that in a deleted neighborhood of the origin, the inverse of the polynomial $\left((i \xi)^{k}+\sum_{m=0}^{k-1} b_{m}(i \xi)^{m}\right)$ is given by the series

$$
(i \xi)^{-1}-\sum_{m=0}^{k-1} b_{m_{1}}(i \xi)^{m_{1}-k}+\cdots+(-1)^{p} \sum_{m_{1}=0}^{k-1} \cdots \sum_{m_{p}=0}^{k-1} b_{m_{1}} \cdots b_{m_{p}}(i \xi)^{m_{1}+\cdots+m_{p}-p k}+\cdots
$$

Upon replacing $(i \xi)^{-L}$ in (10) by $G_{L}(x)$, we reobtain the series (2), which gives us back $E_{b_{0}, \ldots, b_{k-1}}(x)$.

We may also observe that convergence of series (2) does not depend on the vector $x$ belonging to $R^{n}$. Instead, we may assume that $x \in \mathbf{C}^{n}$. It follows that the function $E_{b_{0}}, \ldots, b_{k-1}$ holomorphically extends to a Riemann surface covering $\mathbf{C}^{n} \backslash N(0)$, where $N(0)=\left\{z_{1} e_{1}+\cdots+z_{n} e_{n} \in \mathbf{C}^{n}: z_{1}^{2}+\cdots+z_{n}^{2}=0\right\}$. It follows that any solution to $\left(D_{\mathbf{C}}^{k}+\sum_{m=0}^{k-1} b_{m} D_{\mathbf{C}}^{k}\right) f(z)=0$, where $D_{\mathbf{C}}=\sum_{j=0}^{n} e_{j} \frac{\partial}{\partial z_{j}}$, and $z \in \mathbf{C}^{n}$, in a neighborhood of one of the special manifolds described in [9], automatically has a holomorphic continuation to a covering space of the cells of harmonicity described in [9]. For the particular operator $D_{\mathbf{C}}^{k}+\sum_{m=0}^{k-1} b_{m} D_{\mathbf{C}}^{k}$, this improves upon the holomorphic continuation result given in [9].

We shall now rewrite the operator $D^{k}+\sum_{m=0}^{k-1} b_{m} D^{m}$ as $\left(D-\lambda_{1}\right) \cdots\left(D-\lambda_{k}\right)$, where $\lambda_{1}, \ldots, \lambda_{k}$ are the roots of the polynomial $z^{k}+\sum_{m=0}^{k-1} b_{m} z^{m}$, where $z \in \mathbf{C}$. We shall also rewrite $E_{b_{0}, \ldots, b_{k-1}}$ as $E_{\left(\lambda_{1}, \ldots, \lambda_{k-1}\right)}$.

Proposition 4. $\left(D-\lambda_{1}\right) E_{\left(\lambda_{1}, \lambda_{2}, \ldots, \lambda_{k}\right)}(\underline{x})=E_{\left(\lambda_{2}, \ldots, \lambda_{k}\right)}(x)$.

The result follows by simply inspecting the series (2).

Similarly, we have that $E_{\left(\lambda_{1}, \ldots, \lambda_{k}\right)}(x)(D-\lambda)=E_{\left(\lambda_{2}, \ldots, \lambda_{k}\right)}(x)$. In particular, we have that $(D-\lambda) E_{(\lambda,-\lambda)}(x)=E_{(\lambda)}(x)=E_{(\lambda,-\lambda)}(x)(D-\lambda)$.

Let us now assume that $\lambda$ is real and introduce the function

$$
g_{\lambda}(x, y)=E_{(\lambda,-\lambda)}(y-x)+h(x, y),
$$

where $x, y \in U \times U \backslash\{(x, x): x \in U\}$, and $U$ is a bounded domain with piecewise $C^{1}$, or Lipschitz, boundary. Moreover, the real-valued function $h(x, y)$ satisfies Helmholtz' equation $\left(D^{2}-\lambda^{2}\right) f=0$ in both the variables $x$ and $y$. Using methods described in [4, Chapter 4], and elsewhere, we may arrange it so that $g_{\lambda}(x, y)=0$ on $U \times \partial \operatorname{cl} U$, where $\operatorname{cl} U$ denotes the closure of $U$.

Let us now introduce the function

$$
B_{\lambda}(x, y)=\left(D_{x}+\lambda\right) g_{\lambda}(x, y)\left(D_{y}+\lambda\right)
$$

where $D_{x}=\sum_{j=1}^{n} e_{j} \partial / \partial x_{j}$ and $D_{y}=\sum_{j=1}^{n} e_{j} \partial / \partial y_{j}$. It may be observed that the function $B_{\lambda}(x, y)$ is a continuous function on $U \times U$. By similar arguments to those used in [10], it may be observed that the function $\bar{B}_{\lambda}(x, y)$ 
is the Bergman kernel for $L^{2}$-integrable solution to the equation $(D-\lambda) f=$ 0 on $U$, where for each $a=a_{1}+\cdots+a_{1 \cdots n} e_{1} \cdots e_{n} \in A_{n}(\mathbf{C}), \bar{a}$ denotes $a_{0}+\cdots+(-1)^{1} a_{j_{1} \cdots j_{r}} e_{j_{r}} \cdots e_{j_{1}}+\cdots+(-1)^{n} a_{1 \cdots n} e_{n} \cdots e_{1}$. So, we have that

$$
\int_{U} B(x, y) f(y) d y^{n}=f(x)
$$

for each $x \in U$, whenever $(D-\lambda) f=0$ and $f$ is square integrable over $U$. An explicit formula for $B_{\lambda}(x, y)$ is given in [2] for the case where $U$ is the unit ball in $R^{n}$.

In [10], it is shown that the Bergman kernel for the Dirac operator $D$ is a projection operator from the $A_{n}$-module of $A_{n}$-valued square integrable functions on $U$ to the Bergman $A_{n}$-module of square integrable functions which are solutions to the equation $D f=0$. Similar arguments show that the operator $B_{\lambda}(x, y)$ is also a projection operator.

\section{REFERENCES}

1. F. Brackx, R. Delanghe, and F. Sommen, Clifford analysis, Res. Notes in Math., vol. 76, Pitman, London, 1982.

2. F. Brackx, F. Sommen, and N. Van Acher, Reproducing Bergman kernels in Clifford analysis, Complex Variables 24 (1994), 191-204.

3. A. C. Dixon, On the Newtonian potential, Quart. J. Math. 35 (1904), 283-296.

4. K. Gürlebeck and W. Sprössig, Quaternionic analysis and elliptic boundary value problems, Birkhäuser-Verlag, Basel, 1990.

5. C. Li, A. McIntosh, and S. Semmes, Convolution singular integrals on Lipschitz surfaces, J. Amer. Math. Soc. 5 (1992), 455-481.

6. M. Mitrea, Boundary value problems and Hardy spaces associated to the Helmholtz equation (to appear).

7. W. Rudin, Real and complex analysis, 3rd ed., McGraw-Hill, New York, 1987.

8. J. Ryan, Iterated Dirac operators and conformal transformations in $R^{n}$, Proc. XVth Internat. Conf. on Differential Geometric Methods in Theoretical Physics (H. D. Doebner and J. D. Henning, eds.), World Scientific, Singapore, 1987, pp. 390-399.

9. _ Dirac operators, Schrödinger-type operators in $\mathbf{C}^{n}$, and Huygens' principle, J. Funct. Anal. 87 (1989), 321-347.

10. M. Shapiro and N. Vasilevski, On the Bergman kernel function in hyperholomorphic analysis, Reporte Interno 115 (1993), CINVESTAV del IPN, Mexico (to appear).

11. F. Sommen and X. Zhenyuan, Fundamental solutions for operators which are polynomials in the Dirac operator, Clifford Algebras and Their Applications in Mathematical Physics (A. Micali, R. Boudet, and J. Helmstetter, eds.), Kluwer, Dordrecht, 1992, pp. 313-326.

12. X. Zhenyuan, A function theory for the operator $D-\lambda$, Complex Variables 16 (1991), $37-42$.

Department of Mathematical Sciences, University of Arkansas, Fayetteville, ARKANSAS 72701

E-mail address: jryan@comp.uark.edu 